\title{
Harmonic Maps Between Surfaces and Teichmüller Spaces
}

\author{
Chikako Mese
}

\section{Introduction}

Harmonic maps between surfaces have been important in studies of Teichmüller spaces. In [GR], Gerstenhaber and Rauch proposed constructing the Teichmüller map by a maximum-minimum approach using harmonic maps. This charaterization of the Teichmüller map was further investigated by Reich and Strebel $[\mathbf{R}][\mathbf{R S}]$, Miyahara [Mi], Leite [Le] and Kuwert [Ku]. Furthermore, Wolf [Wo1] [Wo2] [Wo3], Fischer and Tromba $[\mathbf{F T}][\mathbf{T}]$, and S. Yamada $[\mathbf{Y}]$ have used the energy of maps, harmonic with respect to the hyperbolic metric, to study Teichmüller spaces. In this paper, we will study the energy of harmonic maps between surfaces with fixed conformal structures.

It is well known that the energy of maps between surfaces is only dependent on the conformal class of the domain and is independent of the metric in the conformal class. On the other hand, the energy is dependent on the choice of a metric on the target. Fixing a conformal structure on the domain surface and on the target surface, we consider the energy of the energy minimizing map between the surfaces as a function of the metric in the conformal class of the target.

More precisely, let $\Sigma_{1}, \Sigma_{2}$ be Riemann surfaces of genus $s>1$. Let $\mathcal{D}_{\kappa}$ be the space of all conformal metrics $g$ on $\Sigma_{2}$ with curvature bounded from above by $\kappa$ and area equal to 1 . Let $\phi: \Sigma_{1} \rightarrow \Sigma_{2}$ be a homeomorphism. Define

$$
\mathcal{E}: \mathcal{D}_{\kappa} \rightarrow \mathbf{R}
$$

where $\mathcal{E}(g)$ is the energy of the energy minimizing map $f: \Sigma_{1} \rightarrow\left(\Sigma_{2}, g\right)$ in the homotopy class of $\phi$. Now let

$$
\mathcal{E}_{\kappa}=\inf \left\{\mathcal{E}(g): g \in \mathcal{D}_{\kappa}\right\} .
$$

Because energy is always bounded from below by the area, $\mathcal{E}_{\kappa} \geq 1$. We will show that for any $\kappa$, this inequality is strict.

The Gap Theorem. For any $\kappa<\infty, \mathcal{E}_{\kappa}>1$. In particular, if the curvautre of $\left(\Sigma_{2}, g\right)$ is bounded from above, then any continuous map $f: \Sigma_{1} \rightarrow\left(\Sigma_{2}, g\right)$ has energy strictly greater than 1 . On the other hand, the limit of the map $\kappa \mapsto \mathcal{E}_{\kappa}$, as $\kappa \rightarrow \infty$, is equal to 1 .

In proving the above theorem, we will consider metrics on $\Sigma_{2}$ that are not necessarily smooth. A natural class of metrics to consider for this problem are those associated with distance functions of curvature bounded from above in the 
sense of Alexandrov. We will show the following (see Section 2.3 for the meaning of distance functions belonging to a Teichmüller class):

Compactness Theorem for Distance Functions. The space of distance functions of curvature bounded from above by $\kappa$, with area equal to 1 , whose metric topology is equivalent to the surface topology, and belonging to a fixed Teichmüller class is compact under the topology of uniform convergence.

One the fundamental results for harmonic map theory between surfaces is that when the target metric is smooth, the energy minimizing maps are quasiconformal. This result was first obtained by Schoen and Yau with an assumption of non-positive curvature on the target metric and also by Jost and Schoen without the curvature constraint. Harmonic maps fail to be quasiconformal, or even homeomorphic, when the target metric is singular. The collapsing behavior of energy minimizing map was investigated by Kuwert $[\mathbf{K u}]$ for cone metrics and by the author [Me5] for general singular metrics of curvature bounded from above. Here, we will prove that the collapse occurs only in the presence of curvature concentrations. Given a distance function of curvature bounded from above, we define a point to be curvature regular (see precise definition below) if the curvature measure is bounded by the surface measure in its neighborhood. We then have the following theorem:

Univalent HARMONiC MAPs. Let $f: \Sigma_{1} \rightarrow\left(\Sigma_{2}, g\right)$ be an energy minimizing map with respect to a (possibly nonsmooth and degenerate) metric $g$. Suppose $\left(\Sigma_{2}, g\right)$ is a metric space of curvature bounded from above by $\kappa$. If $P \in \Sigma_{2}$ is a curvature regular point, then there exists a neighborhood of $f^{-1}(P)$ in which $f$ is homeomorphic.

This paper is organized as follows: In Section 2, we collect some of the tools needed in this paper; we outline Korevaar and Schoen's Sobolev theory for maps into metric spaces and define what is means for a distance function to be in a certain conformal class. We prove the homeomorphism property of maps when the target curvature is controlled in Section 3 and prove the compactness theorem for distance functions in Section 4. Using these results, we will prove our main result, The Gap Theorem, in Section 5.

\section{Preliminaries}

2.1. Definition of Metric Spaces of Curvature Bounded from Above. First, we review the notion of curvature bounds in a metric space $X$. We assume our metric spaces are length spaces, i.e. for each $P, Q \in X$, there exists a curve $\gamma_{P Q}$ such that the length of $\gamma_{P Q}$ is exactly $d(P, Q)$ (which we will sometimes write as $\left.d_{P Q}\right)$. We call $\gamma_{P Q}$ a geodesic between $P$ and $Q$. We then say that $X$ is an NPC (non-positively curved) space if every point of $X$ is contained in a neighborhood $U$ so that for every $P, Q, R \in U$,

$$
d_{P Q_{\tau}}^{2} \leq(1-\tau) d_{P Q}^{2}+\tau d_{P R}^{2}-\tau(1-\tau) d_{Q R}^{2}
$$

where $Q_{\tau}$ is the point on $\gamma_{Q R}$ so that $d_{Q Q_{\tau}}=\tau d_{Q R}$. Note that equality is achieved for every triplet $P, Q, R \in \mathbf{R}^{2}$. More generally, a length space is said to have curvature bounded from above by $\kappa$ if

$$
\cosh d_{P Q_{\tau}} \leq \frac{\sinh (1-\tau) \kappa d_{Q R}}{\sinh \kappa d_{Q R}} \cosh d_{P Q}+\frac{\sinh \tau \kappa d_{Q R}}{\sinh \kappa d_{Q R}} \cosh d_{P R}
$$


for $\kappa<0$ and

$$
\cos d_{P Q_{\tau}} \geq \frac{\sin (1-\tau) \kappa d_{Q R}}{\sin \kappa d_{Q R}} \cos d_{P Q}+\frac{\sin \tau \kappa d_{Q R}}{\sin \kappa d_{Q R}} \cos d_{P R}
$$

for $\kappa>0$ and $d_{P Q}, d_{Q R}, d_{P R}<\frac{\pi}{\sqrt{\kappa}}$. Note that if $X$ is $S_{\kappa}$, a surface of constant curvature $\kappa$, then the equality is achived for (2) when $\kappa>0$ and for (3) when $\kappa<0$ for every triplet $P, Q, R \in S_{\kappa}$

2.2. Sobolev Space Theory in Complete Metric Spaces. Let $(\Omega, g)$ be a compact Riemannian domain and $(X, d)$ any complete metric space. In $[\mathbf{K S 1}]$ and [KS2], Korevaar and Schoen develop and investigate the space $W^{1,2}(\Omega, X)$. Here we define this space and collect some of their results.

A Borel measurable map $u: \Omega \rightarrow X$ is said to be in $L^{2}(\Omega, X)$ if for $P \in X$,

$$
\int_{\Omega} d^{2}(u(x), P) d z<\infty .
$$

Note that by the triangle inequality, this definition is independent of $P$ chosen. For $u \in L^{2}(\Omega, X)$, we can construct an $\epsilon$-approximate energy function $e_{\epsilon}: \Omega_{\epsilon} \rightarrow \mathbf{R}$ where $\Omega_{\epsilon}=\{x \in \Omega: d(x, \partial \Omega)>\epsilon\}$ by

$$
e_{\epsilon}(x)=\frac{1}{\omega_{n}} \int_{S(x, \epsilon)} \frac{d^{2}(u(x), u(y))}{\epsilon^{2}} \frac{d \sigma}{\epsilon^{n-1}} .
$$

where $\omega_{n}$ is the area form for the unit sphere $S(x, 1)$. For any Borel measure on the interval $(0,2)$ satisfying

$$
\nu \geq 0, \nu((0,2))=1 \text { and } \int_{0}^{2} \lambda^{-2} d \nu(\lambda)<\infty
$$

we can also define approximate an energy density function ${ }_{\nu} e_{\epsilon}(x): \Omega_{2 \epsilon} \rightarrow \mathbf{R}$ by averaging $e_{\epsilon}(x)$. More precisely,

$$
{ }_{\nu} e_{\epsilon}(x)=\int_{0}^{2} e_{\lambda \epsilon}(x) d \nu(\lambda) .
$$

By setting ${ }_{\nu} e_{\epsilon}(x)=0$ for $x \in \Omega-\Omega_{2 \epsilon}$, we can consider ${ }_{\nu} e_{\epsilon}(x)$ to be a $L^{1}$ function on $\Omega$ and hence it defines linear functional $E_{\epsilon}^{u}: C_{c}(\Omega) \rightarrow \mathbf{R}$. We say $u \in L^{2}(\Omega, X)$ has finite energy (or that $u \in W^{1,2}(\Omega, X)$ ) if

$$
E^{u} \equiv \sup _{f \in C_{c}(\Omega), 0 \leq f \leq 1} \limsup _{\epsilon \rightarrow 0} E_{\epsilon}^{u}(f)<\infty .
$$

We say $E^{u}$ is the (Korevaar-Schoen) energy of the map $u$. It can be shown that if $u$ has finite energy, the measures ${ }_{\nu} e_{\epsilon}(x) d x$ converge weakly to a measure, indepently of the choice of $\nu$, which is absolutely continuous with respect to the Lebesgue measure. Therefore, there exists a function $e(x)$, which we call the energy density, so that $e_{\epsilon}(x) d x \rightarrow e(x) d x$. In analogy to the case of real valued functions, we often write $|\nabla u|^{2}(x)$ in place of $e(x)$. In particular,

$$
E^{u}=\int_{\Omega}|\nabla u|^{2} d x
$$

Similarly, the directional energy measures $\left|u_{*}(Z)\right|^{2} d x$ for $Z \in \Gamma \bar{\Omega}$ can also be defined as the weak ${ }^{*}$ limit of measures ${ }^{Z} e_{\epsilon} d x$. Here,

$$
z_{e_{\epsilon}}(x)=\frac{d^{2}(u(x), u(\bar{x}(x, \epsilon))}{\epsilon^{2}} .
$$


where $\bar{x}(x, \epsilon)$ denotes the flow along $Z$ at time $\epsilon$, starting at point $x$. For almost every $x \in \Omega$,

$$
|\nabla u|^{2}(x)=\frac{1}{\omega_{n}} \int_{S^{n-1}}\left|u_{*}(\omega)\right|^{2} d \sigma(\omega)
$$

This definition of Sobolev space $W^{1,2}(\Omega, X)$ is consistent with the usual definition when $X$ is a Riemannian manifold.

The local existence and regularity for energy minimizing maps in this general setting has been worked out by Schoen and Korevaar [KS1] for $\kappa \leq 0$ and by Serbinowski [Se] for $\kappa>0$. A map is called harmonic if it is locally energy minimizing. The homotopy problem for harmonic maps was studied in [KS1]. The regularity theorem can be summarized by the following:

THEOREM 1. If $X$ is a metric space of curvature bounded from above by $\kappa$, $(\Omega, g)$ is a Riemannian domain, and $f: \Omega \rightarrow X$ is a harmonic map, then $f$ is locally Lipzschitz continuous in the interior of $\Omega$. The local Lipschitz constant of $f$ is only dependent on the total energy of $f$, distance to $\partial \Omega$ (if $\partial \Omega \neq \emptyset), \kappa$, and $g$.

Finally, if $X$ has curvature bounded from above by $\kappa$, then for any map $u \in$ $W^{1,2}(\Omega, X)$, we can also make sense of the notion of the pull back metric

$$
\pi: \Gamma(T \bar{\Omega}) \times \Gamma(T \bar{\Omega}) \rightarrow L^{1}(\bar{\Omega})
$$

defined by

$$
\pi(V, W)=\frac{1}{4}\left|u_{*}(V+W)\right|^{2}-\frac{1}{4}\left|u_{*}(V-W)\right|^{2} \text { for } V, W \in \Gamma(T \bar{\Omega}) .
$$

Suppose $\Omega$ is a surface with conformal parameter $z=x+i y$. Then $u \in W^{1,2}(\Omega, X)$ is said to be conformal if

$$
\pi\left(\frac{\partial}{\partial x}, \frac{\partial}{\partial x}\right)=\pi\left(\frac{\partial}{\partial y}, \frac{\partial}{\partial y}\right) \text { and } \pi\left(\frac{\partial}{\partial x}, \frac{\partial}{\partial y}\right)=0 .
$$

$\lambda=\pi\left(\frac{\partial}{\partial x}, \frac{\partial}{\partial x}\right)$ is called the conformal factor of $u$.

Using the Sobolev Space theory outlined above, the author [Me1] [Me2] [Me4] has developed the minimal surface theory in metric spaces of curvature bounded from above by $\kappa$. (See Nikolaev [Ni] for a different approach.) We prove the following two theorems in $[\mathbf{M e 2}]$.

THEOREM 2. Let $\Delta$ be a unit disk in the plane and $u: \Delta \rightarrow(X, d)$ be an energy minimizing map into a metric space of curvature bounded from above by $\kappa$. Then for any $\eta \in C_{c}^{2}(\Delta)$ with $\eta \geq 0$,

$$
\int_{\Delta}|\nabla u|^{2} \triangle \eta \geq-2 \kappa \int_{\Delta} \eta|\nabla u|^{4}
$$

If $u$ is minimal (i.e. also weakly conformal) with conformal factor $\lambda$, then

$$
\int_{\Delta} \lambda \triangle \eta \geq-2 \kappa \int_{\Delta} \eta \lambda^{2}
$$

THEOREM 3. Let $\lambda$ be a conformal factor of the pull back metric under a minimal surface (i.e. a weakly conformal energy minimizing map) $u: \Delta \rightarrow(X, d)$ into metric space of curvature bounded from above by $\kappa$. Then for all non-negative $\varphi \in C_{c}^{\infty}(\Delta)$,

$$
\int_{\Delta} \log \lambda \triangle \varphi \geq-2 \kappa \int_{\Delta} \varphi \lambda
$$


Theorem 2 is a differential inequality satisfied by the energy density function. For smooth harmonic maps, it can be derived by using the Bochner's formula. Recall that the Gaussian curvature of a surface with metric $\lambda|d z|^{2}$ is given by $\frac{-1}{2 \lambda} \triangle \log \lambda$ if $\lambda$ is smooth. Hence the geometric interpretation of Theorem 3 is that the curvature of a minimal surface is bounded from above by the curvature of the ambient space. Furthermore, we have

Proposition 4. For a set $E \subset \Delta$, let $P(E)$ be the perimeter of $E$, i.e. if $\varphi_{E}$ is the characteristic function of the set $E$,

$$
P(E)=\int_{\Delta}\left|\nabla \varphi_{E}\right|=\sup \left\{\int_{\Delta} \varphi_{E} \text { div } g d x: g \in C_{c}^{1}(\Delta),|g(x)| \leq 1\right\} .
$$

Let $E_{\delta}=\{z \in \Delta: \lambda<\delta\}$. Then $P\left(E_{\delta}\right) \rightarrow 0$ as $\delta \rightarrow 0$.

Using Proposition 4 , we can show that line element $\lambda|d z|^{2}$ induces a distance function $d_{\lambda}$ on $\Delta$ whose metric topology is equivalent to the surface topology. It is shown in $[\mathbf{M e 2}]$ that $\left(\Delta, d_{\lambda}\right)$ is a metric space of curvature bounded from above by $\kappa$.

Proof. Since $\lambda$ satisfies inequality (7), $\log \lambda \in W_{l o c}^{1,1}(\Delta)$. By the co-area formula for functions of bounded variations,

$$
\int_{-\infty}^{\infty} \int_{\Delta}\left|\nabla \varphi_{E_{\delta}}\right| d t d x=\int_{\Delta}|\nabla \log \lambda|<\infty
$$

and the result follows immediately.

2.3. Conformal Representation of Surfaces. The following is a theorem of Reshetnyak [Re1].

THEOREM 5. Suppose a compact surface $S$ is endowed with a distance function $d$ which makes $(S, d)$ into metric space of curvature bounded from above by $\kappa$. Assume that the metric topology of $d$ is equivalent to the surface topology. Then for every $P \in S$, there is a neighborhood $U$ of $P$ and a conformal homeomorphism $\psi: \Delta \rightarrow U$ from the unit disk $\Delta$ in the complex plane.

REMARK 6. In [Re1], Theorem 5 is proved by approximating the distance function by those induced from Riemannian metrics. The conformal homeomorphism is then obtained by taking a converging sequence of local corformal maps for the approximating metrics. We have shown in [Me3] that Theorem 5 can be proved using the variational theory of Korevaar and Schoen [KS1] outlined above and the minimal surface theory for metric spaces of curvature bounded from above developed in [Me2]. Although Theorem 5 is proved for the $\kappa=0$ case in [Me3], it is not too difficult to generalize the argument to cover the case when $\kappa>0$.

We also have the following theorem of Huber $[\mathbf{H u}]$ :

THEOREM 7. Let $U_{1}, U_{2} \subset S$ and $\psi_{1}: \Delta \rightarrow U_{1}, \psi_{2}: \Delta \rightarrow U_{2}$ be conformal maps with conformal factors $\lambda_{1}$ and $\lambda_{2}$. If $U_{1} \cap U_{2}$ is non-empty, then the map $T=\psi_{2}^{1} \circ \psi_{1}: \psi_{1}^{-1}\left(U_{1} \cap U_{2}\right) \rightarrow \psi_{2}^{-1}\left(U_{1} \cap U_{2}\right)$ is a conformal map. Moreover,

$$
\lambda_{2}(z)=\left|T^{\prime}(z)\right|^{2} \lambda_{1}(z) \text {. }
$$

The above two theorem gives $(S, d)$ a structure of a Riemann surface. Thus, using the uniformization theorem, there is a Riemann surface $\Sigma$ and a conformal homeomorphism $\iota: \Sigma \rightarrow(S, d)$. 
DEFinition 8. A distance function $d$ on a surface $S$ is said to be in the conformal class of Riemann surface $\Sigma$ if there is a conformal homeomorphism $\iota: \Sigma \rightarrow(S, d)$.

Recall that a point in the Teichmüller space with base surface $S$ is represented by a pair $(\Sigma, h)$ where $\Sigma$ is a Riemann surface and $h: S \rightarrow \Sigma$ is a homeomorphism. Two pairs $(\Sigma, h)$ and $\left(\Sigma^{\prime}, h^{\prime}\right)$ represent the same point if and only if $h^{\prime} \circ h^{-1}$ is homotopic to a holomorphic map.

Definition 9. Let $S$ be a compact surface, $d$ a distance function on $S$ and $(\Sigma, h)$ a point in Teichmüller space with base $S$. Suppose that $(S, d)$ is a metric space of curvature bounded from above by $\kappa$ and the metric topology of $d$ is equivalent to the surface topology. Then $d$ is said to be in the Teichmüller class of $(\Sigma, h)$ if there exists a conformal homeomorphism $\iota: \Sigma \rightarrow(S, d)$ homotopic to $h^{-1}$.

The conformal factor $\lambda$ of $\iota$ gives rise to a quadratic form $g=\lambda|d z|^{2}$ on $\Sigma$ which we call the metric induced by $d$. The quadratic form $g$ induces a distance function $d_{\lambda}$ on $\Sigma$ of curvature bounded from above by $\kappa$. By construction $d$ and $d_{\lambda}$ are infinitesmally isometric; in particular, if $f: \Omega \rightarrow\left(\Sigma, d_{\lambda}\right)$ is a finite energy map, then the energy of the map $f$ is equal to the energy of the map $\iota \circ f: \Omega \rightarrow(S, d)$. Hence, from here on, if $d$ is a distance function on a surface with curvature bounded from above by $\kappa$, we assume that $d$ is defined on the appropriate Riemann surface $\Sigma$ by pulling back $d$ on $S$ to $\Sigma$ by $\iota$. The area of $\Sigma$ with respect to $d$ is given by

$$
A(\Sigma, d)=\int_{\Sigma} \lambda|d z|^{2} .
$$

Using the above definitions, we prove in [Me5] that the energy minimizing map of the homotopy problem can be given as a certain limit of smooth harmonic maps.

THEOREM 10. Let $\Sigma_{1}$ and $\Sigma_{2}$ be Riemann surfaces (with or without boundary) of genus $s$ and let $g_{0}$ be a (possibly non-smooth and degenerate) conformal metric which induces a metric space $\left(\Sigma_{2}, d_{0}\right)$ of curvature bounded from above by $\kappa$. Let $h: \Sigma_{1} \rightarrow \Sigma_{2}$, be a degree 1 map. There exists smooth conformal metrics $g_{i}$, $i=1,2, \ldots$ (with induced distance functions $d_{i}$ ) on $\Sigma_{2}$ and maps $f^{i}: \Sigma_{1} \rightarrow \Sigma_{2}$, $i=0,1,2, \ldots$, energy minimizing in the homotopy class of $h$ (with $f^{i}=h$ on $\partial \Sigma_{1}$ if $\left.\partial \Sigma_{2} \neq \emptyset\right)$ with respect to $g_{i}$ so that $(i) d_{i}\left(f^{i}(\cdot), f^{i}(\cdot)\right)$ converges to $d_{0}\left(f^{0}(\cdot), f^{0}(\cdot)\right)$ uniformly on $\Sigma_{2} \times \Sigma_{2}$ and (ii) the (Korevaar-Schoen) energy and the directional energy of $f^{i}$ with respect to the $d_{i}$ converge to that of $f^{0}$ with respect to $d_{0}$.

Let $\lambda_{h}|d z|^{2}$ be the local expression of the hyperbolic metric and let $\rho$ be the function on $\Sigma$ defined by $\lambda|d z|^{2}=\rho \lambda_{h}|d z|^{2}$. By Theorem 3 and using the fact that $\triangle \log \lambda_{h}=2 \lambda_{h}$, we deduce

$$
\int(\triangle \varphi) \log \rho|d z|^{2} \geq-2 \kappa \int \varphi \rho \lambda_{h}|d z|^{2}-2 \int \varphi \lambda_{h}|d z|^{2}
$$

for every $\varphi \in C_{c}^{\infty}(\Delta)$. Equivalently, we can write

$$
\triangle_{g} \log \rho \geq-2 \kappa \rho \lambda_{h}-2 \lambda_{h} \quad \text { weakly. }
$$

We call $\rho$ the function associated with $d$. 


\section{Univalent Harmonic Maps Between Surfaces}

The following is a theorem of Jost and Schoen:

TheOREM 11. Let $\Sigma_{1}$ and $\Sigma_{2}$ be closed Riemann surfaces and $g$ be a smooth Riemannian metric on $\Sigma_{2}$. If $f: \Sigma_{1} \rightarrow\left(\Sigma_{2}, g\right)$ is a degree 1 energy minimizing map, it is a diffeomorphism.

Harmonic maps that are not smooth exhibit behavior that is quite different than the one displayed by smooth harmonic maps; as indicated in [Ku] and [Me5], degree 1 energy minimizing map $f$ between compact surfaces may collapse along vertical arcs of the Hopf differential when $S$ contains singularities. In fact, when the target has a flat metric with cone singularities, the only homeomorphic energy minimizing map is the Teichmüller map with the Teichüller metric on $S$ (see [Le] and $[\mathbf{K u}])$. On the other hand, these energy minimizing maps into surfaces with cone singularities are local homeomorphisms in neighborhoods mapped away from cone points. In this section, we study the behavior of an energy minimizing map $f: \Sigma_{1} \rightarrow\left(\Sigma_{2}, g\right)$ where $g$ is a singular metric and $f$ is mapped to neighborhoods of $\Sigma_{2}$ not containing negative curvature concentrations.

Let $S, \Sigma, d, \iota$, and $\lambda$ as in the Section 2.2. Since $\iota$ is an energy minimizing map, $\lambda$ can be assumed to be bounded above by $L$ locally by Theorem 1 . Therefore, by Theorem 3 ,

$$
\triangle \log \lambda \geq-2 \kappa L \text { weakly }
$$

in a coordinate neighborhood of $\Sigma$. By the Reisz representation of subharmonic functions (see $[\mathbf{H K}]$ ), $\lambda$ can be represented locally by

$$
\lambda(w)=\exp \frac{1}{2}\left(\int \log |w-\xi| d \mu_{1}(\xi)-\int \log |w-\xi| d \mu_{2}(\xi)+h(w)\right)
$$

where $\mu_{1}$ and $\mu_{2}$ are positive measures and $h$ is a harmonic function. Let $\mathcal{K}=$ $\mu_{1}-\mu_{2}$. By the Hahn Decomposition Theorem, there exists two mutually singular positive measures $\mathcal{K}_{+}$and $\mathcal{K}_{-}$so that $\mathcal{K}=\mathcal{K}_{-}-\mathcal{K}_{+}$. If $\lambda|d z|^{2}$ is a smooth metric, the curvature of $\Sigma_{2}$ with respect to the $\lambda|d z|^{2}$ is given by

$$
-K=-\frac{1}{2 \lambda} \triangle \log \lambda .
$$

Hence, the measure $\mathcal{K}$ is the curvature measure for $\lambda|d z|^{2}$. In other words, for any $E \subset \Sigma_{2}$,

$$
\mathcal{K}(E)=\int_{E} \triangle \log \lambda|d w|^{2} .
$$

Since the $\lambda|d z|^{2}$ is not necessarily smooth and may be degenerate, the curvature measure $\mathcal{K}$ could be quite singular. We study neighborhoods in $\Sigma_{2}$ at which the curvature measure satisfies some regularity condition.

Definition 12. A point $w_{0} \in \Sigma_{2}$ is called a curvature regular point if there exists a neighborhood $U$ of $w_{0}$ in which

$$
d \mathcal{K} \leq C|d w|^{2},
$$

for some constant $C$. 
Let $\log \lambda$ be represented as in equation (10) in a compact set $E$ and $\Delta(w)$ be the unit disk centered at $w$. Then

$$
\begin{aligned}
\log \lambda(w) & \geq \frac{1}{2} \int_{\Delta(w) \cap E} \log |w-\xi| d \mathcal{K}+h(w) \\
& \geq \frac{C}{2} \int_{0}^{2 \pi} \int_{0}^{1} \log r r d r d \theta+h(w) \\
& \geq C \pi \int_{0}^{1} \log r r d r+h(w) \\
& \geq-\frac{C \pi}{2}+h(w) .
\end{aligned}
$$

Hence, $\lambda$ is locally uniformly bounded away from zero in a small compact subset of a curvature regular set.

Let $D_{2} \subset \Sigma_{2}$ and $g=\lambda|d z|^{2}$ in $D_{2}$. When we refer to the euclidean metric, we will mean $D_{2}$ with flat metric $|d z|^{2}$. Now suppose that $d \mathcal{K} \leq C|d w|^{2}$ in $D_{2}$. From the above discussion, we may assume that $0<\epsilon \leq \lambda \leq \epsilon^{-1}$ in $D_{2}$. Let $D_{1} \subset \Sigma_{1}$ be a coordinate neighborhood so that $f\left(D_{1}\right) \subset D_{2}$.

For $w_{1}, w_{2} \in D_{2}$, let $\gamma$ be a smooth curve so that

$$
2 d\left(w_{1}, w_{2}\right) \geq \int_{\gamma} \sqrt{\lambda} d s .
$$

Then

$$
d\left(w_{1}, w_{2}\right) \geq \frac{1}{2} \int_{\gamma} \sqrt{\lambda} d s \geq \frac{\epsilon}{2} \operatorname{length}(\gamma) \geq \frac{\epsilon}{2}\left|w_{1}-w_{2}\right|_{\text {eucl }}
$$

where length $(\gamma)$ is the euclidean length of $\gamma$ and $|\cdot|_{\text {eucl }}$ is the euclidean distance. This implies that $f$, which is Lipschitz with respect to $d$, is also Lipschitz in $D_{1}$ with respect to the euclidean coordinates on $D_{2}$. If $f$ has this property, we will say that $f$ is Lipschitz in $D_{1}$ with respect to the flat metric. By Radamacher's Theorem, $f$ is differentiable a.e. and $f_{z}$ and $f_{\bar{z}}$ are defined a.e. Therefore, we can define the stretch factor $k(z)$ for the map $f$.

Definition 13. Let $f: \Sigma_{1} \rightarrow \Sigma_{2}$ be an energy minimizing map. Suppose $D_{2}$ contains only curvature regular points. Let $D_{1} \subset \Sigma_{1}$ so that $f\left(D_{1}\right) \subset D_{2}$. Then the stretch factor of $f$ in $D_{1}$ is defined by

$$
k(z)=\frac{\left|f_{\bar{z}}\right|^{2}}{\left|f_{z}\right|^{2}}
$$

whenever $f_{z}$ and $f_{\bar{z}}$ exists (see $[\mathbf{A h}]$ ).

In our situation, we can prove the following property of $k(z)$.

LEMMA 14. The function $k(z)$ is lower semicontinuous.

Proof. ¿From the proof of Lemma 5 of $[\mathbf{K u}], k\left(z_{0}\right)=\mu-\sqrt{\mu^{2}-1}$ where

$$
\mu=\lim _{\sigma \rightarrow 0} \frac{\int_{\partial D_{\sigma}} d^{2}\left(f(z), f\left(z_{0}\right)\right) d \Sigma}{\pi \sigma^{m+3}}
$$

Since $\mu$ is a non-increasing limit of continuous functions, $\mu$ is upper semicontinuous. It follows that $k$ is lower semicontinuous. 
Let $g_{i}=\lambda_{i}|d z|^{2}$ and $f^{i}$ for $i=0,1,2, \ldots$ as in Theorem 10 with $\Sigma_{1}=D_{1}$. It is a result of citeserbinowski that the Dirichlet solution in a metric space of curvature bounded from above by $\kappa$ is unique as long as the boundary data lies in a small geodesic ball of the target. Thus if we choose $D_{1}$ sufficiently small, $f^{0}$ is equal to $f$ in $D_{1}$. Thus, $f^{i}$ converges to $f$ in the sense of Theorem 10. Let ${ }^{g_{i}} E^{f^{i}}\left(D_{1}\right)$ be the energy of the map $f^{i}$ with respect to the metric $g_{i}$ in the domain $D_{1}$. Then ${ }^{g_{i}} E^{f^{i}}\left(D_{1}\right)$ is uniformly bounded. Since $0<\epsilon \leq \lambda \leq \epsilon^{-1}$, the maps $f^{i}$ are uniformly Lipschitz continuous with respect to the flat metric. Thus, we can assume (by taking a subsequence if necessary) that the sequence $\left\{f^{i}\right\}$ converges uniformly and $H^{1}$ weakly to $f$ with respect to the flat metric. We will write $f^{i}(z)=\left(u^{i}(z), v^{i}(z)\right)$ and $f(z)=(u(z), v(z))$ in local coordinates. Note that the weak convergence of $\frac{\partial u^{i}}{\partial x}$ to $\frac{\partial u}{\partial x}$ in $L^{2}$ implies that $\frac{\partial u^{i}}{\partial x}$ converges a.e. to $\frac{\partial u}{\partial x}$. Similarly, we have this property for $\frac{\partial v^{i}}{\partial x}, \frac{\partial u^{i}}{\partial y}, \frac{\partial v^{i}}{\partial y}$ and hence for $f_{z}^{i}$ and $f_{\bar{z}}^{i}$. Furthermore, let $\mathcal{K}_{i}$ be a function on defined $D_{1}$ by setting $K_{i}(z)$ as $\frac{-1}{2 \lambda_{i}} \triangle \log \lambda_{i}$ at $f(z)$. Let $d \mathcal{K}_{i}$ be the curvature curvature measure of $g_{i}$ defined on $D_{2}$. The curvature measures $\mathcal{K}_{i}$ converges weakly to $\mathcal{K}$ since $\frac{\epsilon}{2} \leq \lambda_{i} \leq 2 \epsilon^{-1}$. Thus, we can assume $d \mathcal{K}_{i} \leq C|d w|^{2}$.

Lemma 15. Assume $z_{0} \in \Sigma_{1}$ so that $k\left(z_{0}\right)=1$ and $f\left(z_{0}\right)$ is a curvature regular point. Then there is a coordinate neighborhood $D_{1}$ around $z_{0}$ so that for some $C_{1}$,

$$
\int_{D_{1}} \triangle \varphi \log k^{-1} \leq C_{1} \int_{D_{1}} \varphi \log k^{-1}
$$

for every $\varphi \in C_{c}^{\infty}(U)$.

Proof. For any $\delta>0, k(z)>1-\delta$ in a sufficiently small neighborhood of $z_{0}$ since $k$ is lower semicontinuous by Lemma 14 . Hence given $C_{2}>1$, there is a sufficiently small neighborhood of $z_{0}$ so that,

$$
k^{-1}-1 \leq C_{2} \log k^{-1} .
$$

Therefore, if $D_{1}$ and $D_{2}$ are defined as in the above discussion, we can arrange them so that inequality (12) holds in $D_{1}$ and $d \mathcal{K} \geq C|d w|^{2}$ in $D_{2}$.

Since $f^{i}$ is a smooth energy minimizing map,

$$
\triangle \log \frac{\left|f_{z}^{i}\right|^{2}}{\left|f_{\bar{z}}^{i}\right|^{2}}=-4 K_{i} \lambda_{i}\left(\left|f_{z}^{i}\right|^{2}-\left|f_{\bar{z}}^{i}\right|^{2}\right),
$$

by $[\mathbf{S Y}]$. Noting that $\left|f_{z}^{i}\right|^{2}-\left|f_{\bar{z}}^{i}\right|^{2}$ is the Jacobian of $f$,

$$
\begin{aligned}
-4 \int_{D_{1}} \varphi K_{i} \lambda_{i}\left(\left|f_{z}^{i}\right|^{2}-\left|f_{\bar{z}}^{i}\right|^{2}\right)|d z|^{2} & =2 \int_{D_{2}}\left(\varphi \circ\left(f^{i}\right)^{-1}\right) \triangle \log \lambda_{i}|d w|^{2} \\
& =2 \int_{D_{2}}\left(\varphi \circ\left(f^{i}\right)^{-1}\right) d \mathcal{K}_{\sigma_{i}} \\
& \leq 2 C \int_{D_{2}} \varphi \circ\left(f^{i}\right)^{-1}|d w|^{2} \\
& =2 C \int_{D_{1}} \varphi\left(\left|f_{z}^{i}\right|^{2}-\left|f_{\bar{z}}^{i}\right|^{2}\right)|d z|^{2}
\end{aligned}
$$

Since $f_{z}^{i} \rightarrow f_{z}, f_{\bar{z}}^{i} \rightarrow f_{\bar{z}}$ a.e. and $\left|f_{z}^{i}\right|,\left|f_{\bar{z}}^{i}\right| \leq L$, by the Lebesgue Convergence Theorem,

$$
C \int_{D_{1}} \varphi\left(\left|f_{z}^{i}\right|^{2}-\left|f_{\bar{z}}^{i}\right|^{2}\right)|d z|^{2} \rightarrow C \int_{D_{1}} \varphi\left(\left|f_{z}\right|^{2}-\left|f_{\bar{z}}\right|^{2}\right)|d z|^{2}
$$


Furthremore,

$$
\begin{aligned}
C \int_{D_{1}} \varphi\left(\left|f_{z}\right|^{2}-\left|f_{\bar{z}}\right|^{2}\right)|d z|^{2} & \leq C \int_{D_{1}} \varphi\left|f_{\bar{z}}\right|^{2}\left(k^{-1}-1\right)|d z|^{2} \\
& \leq C C_{2} L^{2} \int_{D_{1}} \varphi \log k^{-1}|d z|^{2}
\end{aligned}
$$

On the other hand, $\log \frac{\left|f_{z}^{i}\right|^{2}}{\left|f_{\bar{z}}^{i}\right|^{2}} \rightarrow \log \frac{\left|f_{z}\right|^{2}}{\left|f_{\bar{z}}\right|^{2}}$ a.e. and

$$
\begin{aligned}
0 & \leq \int_{D_{1}} \varphi \triangle \log \frac{\left|f_{z}^{i}\right|^{2}}{\left|f_{\bar{z}}^{i}\right|^{2}}|d z|^{2} \\
& =\int_{D_{1}}(\triangle \varphi) \log \frac{\left|f_{z}^{i}\right|^{2}}{\left|f_{\bar{z}}^{i}\right|^{2}}|d z|^{2} \\
& \leq C \int_{D_{2}} \varphi d \mathcal{K}_{\sigma_{i}} .
\end{aligned}
$$

By the Lebesgue Dominated Convergence Theorem,

$$
\int_{D_{1}} \triangle \varphi \log \frac{\left|f_{z}^{i}\right|^{2}}{\left|f_{\bar{z}}^{i}\right|^{2}}|d z|^{2} \rightarrow \int_{D_{1}} \triangle \varphi \log \frac{\left|f_{z}\right|^{2}}{\left|f_{\bar{z}}\right|^{2}}|d z|^{2}
$$

We are done by letting $C_{1}=2 C \cdot C_{2} \cdot L^{2}$.

TheOREM 16. Let $\phi: \Sigma_{1} \rightarrow \Sigma_{2}$ be a homeomorphism. Let $f: \Sigma_{1} \rightarrow \Sigma_{2}$ be energy minimizing in the homotopy class of $\phi$ with respect to a (possibly nonsmooth and degenerate) metric $g$. Suppose $\left(\Sigma_{2}, g\right)$ is a metric space of curvature bounded from above by $\kappa$ and $U \subset \Sigma_{2}$ contains only curvature regular points. Then $f$ is a local homeomorphism in $f^{-1}(U)$.

Proof. Suppose $f$ is not a local homeomorphism in $f^{-1}(U)$. Then by [Me5], $f$ collapses a vertical arc of its Hopf differential and for some $z \in U, k(z)=1$. It is well known that the differential inequality (11) implies that the infimum of $k^{-1}$ in a ball of radius $\frac{r}{2}$ is bounded below by a constant times its average value on the concentric ball of radius $r$ for $r<1$ (see for example [Mo]). Hence, if $k=1$ then at a point, then $k \equiv 1$ in its neighborhood. But this is a contradiction since the collapse occurs only along the vertical arcs of the Hopf differential of $f$.

This immediately yields the following generalization of $[\mathbf{S Y}]$.

COROLlary 17. Let $f: \Sigma_{1} \rightarrow \Sigma_{2}$ be energy minimizing with respect to a (possibly nonsmooth) metric $g$. Suppose $\left(\Sigma_{2}, g\right)$ is a metric space of curvature bounded from above by $\kappa$ and every point of $\Sigma_{2}$ is curvature regular, then there exists a homeomorphic energy minimizing map $f: \Sigma_{1} \rightarrow \Sigma_{2}$.

Proof. By Theorem 10, there exists an energy minimizing map $f$ which is a limit of smooth energy minimizing maps. By Theorem 16, $f$ is a local homeomorphism and hence a global homeomorphism.

\section{Compactness Theorem for Distance Functions}

Let $(S, h)$ be a point in Teichmüller space. Let $\mathcal{D}_{\kappa}$ be a space of all distance functions on $d$ of curvature bounded above by $\kappa$, area equal to 1 , and in the Teichmüller class of $(\Sigma, h)$. For $d \in \mathcal{D}_{\kappa}$, let $\iota: \Sigma \rightarrow(S, d)$ be the conformal map 
homotopic to $h^{-1}$ with conformal factor $\lambda$. We can indentify $\mathcal{D}_{\kappa}$ with the space of metrics $g=\lambda|d z|^{2}$ on $\Sigma$ induced by $d$. Since $\iota$ is a conformal map, its total energy is equal to the area. Hence for any $d$, its conformal factor $\lambda=|\nabla \iota|^{2}$ is bounded (where the bound only depends on $\kappa$ since total energy is equal to 1 for any $d \in \mathcal{D}_{\kappa}$ ) by Theorem 1 . Hence we let $\lambda<\epsilon^{-1}$ for some $\epsilon>0$.

We prove the following compactness theorem for $\mathcal{D}_{\kappa}$.

TheOREM 18. Let $\left\{d_{i}\right\}$ be a sequence of distance function in $\mathcal{D}_{\kappa}$. Then there exists a subsequence of $\left\{d_{i}\right\}$ converging uniformly to a distance function in $\mathcal{D}_{\kappa}$.

Proof. Let $d \in \mathcal{D}_{\kappa}$ and let $\iota: \Sigma \rightarrow(S, d)$ as above. Whenever convenient, we will identify $S$ and $\Sigma$ by $\iota$. We work in a coordinate neighborhood $\Delta \subset S$. Let $\left(w_{1}, w_{2}\right),\left(\xi_{1}, \xi_{2}\right) \in \Delta \times \Delta$, we have

$$
\begin{aligned}
\left|d\left(w_{1}, w_{2}\right)-d\left(\xi_{1}, \xi_{2}\right)\right| & \leq d\left(w_{1}, \xi_{1}\right)+d\left(w_{2}, \xi_{2}\right) \\
& \leq \int_{\gamma_{1}} \sqrt{\lambda} d s+\int_{\gamma_{2}} \sqrt{\lambda} d s \\
& \leq \epsilon^{-1}\left(\left|w_{1}-\xi_{1}\right|+\left|w_{2}-\xi_{2}\right|\right) \\
& \leq 2 \epsilon^{-1} \sqrt{\left|w_{1}-\xi_{1}\right|^{2}+\left|w_{2}-\xi_{2}\right|^{2}}
\end{aligned}
$$

where $\gamma_{i}$ is the Euclidean lines between $w_{i}$ and $\xi_{i}$.

Hence $\mathcal{D}_{\kappa}$ is an equicontinuous family of functions on $\Delta \times \Delta$ and there exists a subsequence of $\left\{d_{i}\right\}$ converging uniformly. Since $S$ is compact, there exists a subsequence of $\left\{d_{i}\right\}$ (which we still denote $\left\{d_{i}\right\}$ by an abuse of notation) converging uniformly, say to a function $d: S \times S \rightarrow \mathbf{R}$.

Now we must show that $d$ is an distance function of curvature bounded from above by $\kappa$ and $d$ is in the Teichmüller class of $(S, h)$. Let $\iota_{i}: \Sigma \rightarrow\left(S, d_{i}\right)$ be a conformal homeomorphism homotopic to $h^{-1}$. (Note that we can also think of $\iota$ as the identity map if we assume that $d_{i}$ is defined on $\Sigma$.) By Theorem 10, there is an energy minimizing map $\iota: \Sigma \rightarrow(S, d)$ and a subsequence of $\left\{\iota_{i}\right\}$ so that the Sobolev and directional energies converges weakly to that of $\iota$. Because $\left\{\iota_{i}\right\}$ is a sequence of conformal maps, $\iota$ is also conformal. Let $\lambda_{i}$ and $\lambda$ be the conformal factors of $\iota_{i}$ and $\iota$ respectively. Furthermore, let $\iota_{i, \epsilon}: \Delta \rightarrow\left(S \times \Delta, d_{i, \sigma}\right)$ be defined by $\iota_{i, \sigma}(z)=\left(\iota_{i} \circ \phi, z\right)$ and where $d_{i, \sigma}^{2}=d_{i}^{2}+\sigma^{2}|z|^{2}$. Then $\iota_{i, \sigma}$ is a conformal map with conformal factor $\lambda_{i, \sigma}=\sqrt{\lambda^{2}+\sigma}$. By Theorem 3

$$
\int \triangle \varphi \log \lambda_{i, \sigma} \geq 2 \kappa \int \varphi \lambda_{i, \sigma}
$$

Since the energy of $\iota_{i}$ uniformly bounded, $\lambda_{i}$ is locally uniformly (i.e. independently of $i$ ) bounded and hence we assume $\lambda_{i} \leq \epsilon^{-1}$ in supp $\varphi$. Hence $\log \sigma^{2} \leq \log \lambda_{i, \sigma} \leq \log \left(\epsilon^{-1}+\sigma^{2}\right)$ in supp $\varphi$. By the Lebesque Dominated Convergence Theorem,

$$
\int \triangle \varphi \log \lambda_{\sigma} \geq 2 \kappa \int \varphi \lambda_{\sigma}
$$

as $i \rightarrow \infty$. Now let $\sigma \rightarrow 0$ and we see that

$$
\int \triangle \varphi \log \lambda \geq 2 \kappa \int \varphi \lambda
$$


by Lemma 5.5 of $[\mathbf{M e 2}]$. By Theorem 6.1 of $[\mathbf{M e 2}]$, this inequality shows that the line element $\sqrt{\lambda}|d z|^{2}$ induces a distance function $d_{\lambda}$ whose metric topology is equivalent to the surface topology and that its curvature is bounded above by $\kappa$. This then shows that $d$ has the desired properties.

\section{The Minimum Energy for Maps between Two Surfaces}

5.1. The Variational Problem. We now describe our variational problem. Let $\Sigma_{1}$ and $\Sigma_{2}$ be Riemann surfaces of genus $s>1$ and $\phi: \Sigma_{1} \rightarrow \Sigma_{2}$ be a homeomorphism. Let $S$ be a surface of genus 1 and let $\mathcal{D}_{\kappa}$ as in Section 4 with $\Sigma=\Sigma_{2}$ and $h=\phi$. Recall that each $d \in \mathcal{D}_{\kappa}$ induces a metric $g=\lambda|d z|^{2}$ on $\Sigma_{2}$ and $g$ can be written as $g=\rho g_{h}$ where $g_{h}=\lambda_{h}|d z|^{2}$ is the hyperbolic metric and $\rho: \Sigma_{2} \rightarrow \mathbf{R}$ satisfies inequality 9. We will call $\rho$ the function associated with $d$. Again, we identify $\mathcal{D}_{\kappa}$ with the space of metrics $g$ induced by $d \in \mathcal{D}_{\kappa}$.

We define the function $\mathcal{E}: \mathcal{D}_{\kappa} \rightarrow \mathbf{R}$ by

$$
\mathcal{E}(d)={ }^{d} E^{f}
$$

where ${ }^{d} E^{f}$ is the (Korevaar-Schoen) energy of the energy minimizing map $f: \Sigma_{1} \rightarrow$ $\Sigma_{2}$ in the homotopy class of $\phi$ with respect to $d \in \mathcal{D}_{\kappa}$. Let

$$
\mathcal{E}_{\kappa}=\inf _{d \in \mathcal{D}_{\kappa}} \mathcal{E}(d)
$$

TheOREM 19. There exists $d \in \mathcal{D}_{\kappa}$ so that $\mathcal{E}_{\kappa}=\mathcal{E}(d)$.

Proof. Let $\left\{d_{i}\right\} \subset \mathcal{D}_{\kappa}$ be a minimizing sequence for $\mathcal{E}$ in $\mathcal{D}_{\kappa}$. By Theorem 18, we can choose a subsequence of $\left\{d_{i}\right\}$ (which we still denote by $\left\{d_{i}\right\}$ ) so that $d_{i}$ converges uniformly, say to $d \in \mathcal{D}_{\kappa}$. Let $f_{i}: \Sigma_{1} \rightarrow \Sigma_{2}$ be the energy minimizing map with respect to distance function $d_{i}$ homotopic to $\psi$. Since $\left\{d_{i}\right\}$ is a minimizing sequence for $\mathcal{E}: \mathcal{D}_{\kappa} \rightarrow \mathbf{R}$, the energy of $f_{i}$ (with respect to $d_{i}$ ) is bounded indenpendently of $i$. Hence by Theorem 15 of [Me5], there exists a map $f$, energy minimizing with respect to $d$, and a subsequence of $\left\{f_{i^{\prime}}\right\}$ so that the Sobolev energies converge to that of $f$. Hence,

$$
\mathcal{E}_{\kappa} \leq{ }^{g} E^{f}=\lim _{i^{\prime} \rightarrow \infty}{ }^{g_{i}} E^{f_{i^{\prime}}}=\mathcal{E}_{\kappa} .
$$

Hence $\mathcal{E}_{\kappa}=\mathcal{E}(d)$.

We call $(d, f)$ the minimizer for $\kappa$ if the energy with respect to $d \in \mathcal{D}_{\kappa}$ of the energy minimizer $f$ homotopic to $\phi$ achieves the minimum value of $\mathcal{E}_{\kappa}$. Since the energy of a map is always bounded from below by the area, $\mathcal{E}_{\kappa} \geq 1$.

5.2. The First Variation Argument. We will investigate the behavior of a minimizer $(d, f)$ for $\kappa$. To do so, we use the first variation argument which we describe below.

Let $\Delta$ be a unit disk on the plane. We will say that a map $f: \Delta \rightarrow \Sigma_{2}$ is Lipschitz with respect to the flat metric if there is a coordinate chart $\psi: \Delta \rightarrow$ $f(\Delta) \subset \Sigma_{2}$ so that $\psi^{-1} \circ f: \Delta \rightarrow \Delta$ is Lipschitz with respect to the standard euclidean coordinate on $\Delta$. 
LEMma 20. Let $g=\rho|d z|^{2}$ be a metric induced by a distance function $d \in \mathcal{D}_{\kappa}$. If $f: \Delta \rightarrow\left(\Sigma_{2}, g\right)$ is a finite energy map and $\rho \geq \epsilon>0$ in $f(\Delta)$ then the (KorevaarSchoen) energy of $f$ with respect to $d$ can be written

$$
{ }^{d} E^{f}=\int_{\Delta} \lambda\left(\left|\frac{\partial f}{\partial x}\right|^{2}+\left|\frac{\partial f}{\partial y}\right|^{2}\right) .
$$

Proof. Let $g_{i}=\lambda_{i}|d z|^{2}$ and $f^{i}$ as in Theorem 10 and let $d_{i}$ be the distance function associated with $g_{i}$. By the paragraph preceeding Lemma ??, we see that $\left\{f^{i}\right\}$ is uniformly Lipschitz in $\Delta$ with respect to the flat metric and

$$
\left|\frac{\partial f^{i}}{\partial x}\right| \rightarrow\left|\frac{\partial f}{\partial x}\right|, \quad\left|\frac{\partial f^{i}}{\partial y}\right| \rightarrow\left|\frac{\partial f}{\partial y}\right|, \quad \lambda_{i} \rightarrow \lambda
$$

and ${ }^{d} E^{f}=\lim _{1 \rightarrow \infty}{ }^{d_{i}} E^{f^{i}}$. Since $g_{i}$ is a smooth metric,

$$
{ }^{d_{i}} E^{f^{i}}=\int_{\Delta} \lambda_{i}\left(\left|\frac{\partial f^{i}}{\partial x}\right|^{2}+\left|\frac{\partial f^{i}}{\partial y}\right|^{2}\right) .
$$

By the Lebesgue Convergence Theorem, letting $i \rightarrow \infty$,

$$
{ }^{d} E^{f}=\int_{\Delta} \lambda\left(\left|\frac{\partial f}{\partial x}\right|^{2}+\left|\frac{\partial f}{\partial y}\right|^{2}\right) .
$$

Suppose $d, \tilde{d} \in \mathcal{D}_{\kappa}$ and let $g$ and $\tilde{g}$ be the metrics induced from $d$ and $\tilde{d}$ respectively. Furthermore, assume that $g$ and $\tilde{g}$ only differs from each other in a coordinate neighborhood $\Delta \subset \Sigma_{2}$. Assume $f$ is of finite energy with respect both metrics and let $g=\rho \lambda_{h}|d z|^{2}, \tilde{g}=\tilde{\rho} \lambda_{h}|d z|^{2}$ and $\eta=\sqrt{\rho \tilde{\rho}}$. Define

$$
\rho_{t}=\frac{(1-t) \rho^{2}+t \eta^{2}}{\int_{\Sigma}\left((1-t) \rho^{2}+t \eta^{2}\right) d \mu_{g}}
$$

and let $d_{t}$ be the distance function associated to $g_{t}=\rho_{t} \lambda_{h}|d z|^{2}$. We see that

$$
\dot{\rho}_{t}=\frac{1}{2}\left(\frac{\eta^{2}}{\rho}-\rho\left(\int \frac{\eta^{2}}{\rho}\right)\right)=\frac{1}{2}(\tilde{\rho}-\rho) .
$$

We also assume $f$ is Lipschitz with respect to the flat metric on $f^{-1}(\Delta) \subset \Sigma_{1}$. Since $f$ is of finite energy with respect to both $g$ and $\tilde{g}$,

$$
\int_{f^{-1}(\Delta)} \rho_{t} \lambda_{h}\left(\left|\frac{\partial f}{\partial x}\right|^{2}+\left|\frac{\partial f}{\partial y}\right|^{2}\right)|d z|^{2}<\infty
$$

and

$$
\begin{aligned}
& \left.\int_{f^{-1}(\Delta)}\left(\frac{d}{d t} \rho_{t}\right)\right|_{t=0} \lambda_{h}\left(\left|\frac{\partial f}{\partial x}\right|^{2}+\left|\frac{\partial f}{\partial y}\right|^{2}\right)|d z|^{2} \\
& \quad=\frac{1}{2} \int_{f^{-1}(\Delta)} \tilde{\rho} \lambda_{h}\left(\left|\frac{\partial f}{\partial x}\right|^{2}+\left|\frac{\partial f}{\partial y}\right|^{2}\right)|d z|^{2}-\frac{1}{2} \int_{f^{-1}(\Delta)} \rho \lambda_{h}\left(\left|\frac{\partial f}{\partial x}\right|^{2}+\left|\frac{\partial f}{\partial y}\right|^{2}\right)|d z|^{2}
\end{aligned}
$$

If $(d, f)$ is a minimizer for $\kappa$, then $\left.\frac{d}{d t} d_{t} E^{f}\right|_{t=0}=0$. Thus, by the calculation above, $\tilde{d}^{\tilde{d}} E^{f}={ }^{d} E^{f}$ and $(\tilde{d}, f)$ is also a minimizer for $\kappa$. 
5.3. The Gap Property. In this section, we prove our main theorem which implies that if a metric $g$ on the target surface $\Sigma_{2}$ has curvature bounded from above, then the energy of any continuous map $f: \Sigma_{1} \rightarrow \Sigma_{2}$ with respect to $g$ is strictly greater than the area of $\Sigma_{2}$ with respect to $g$.

THEOREM 21. For every $\kappa$, there exists $\tilde{\kappa}>\kappa$ so that $\mathcal{E}_{\kappa}>\mathcal{E}_{\kappa^{\prime}}$ for every $\kappa^{\prime}>\tilde{\kappa}$. In particular, for every $\kappa, \mathcal{E}_{\kappa}>1$.

Proof. Let $(d, f)$ be a minimizer for $\kappa$ and let $g=\rho \lambda_{h}|d z|^{2}$ be the metric induced by $d$. By Proposition 4 , there exists a point $w_{0} \in \Sigma_{2}$ so that $\rho \geq \epsilon>0$ in a neighborhood of $w_{0}$. Take a coordinate neighborhood $\Delta \subset \Sigma_{2}$ of $w_{0}$ and let $\lambda=\rho \lambda_{h}$ in $\Delta$. Assume $\Delta$ is a disk of radius 1 , the point $w_{0}$ corresponds to the origin $O$ of $\Delta$, and $\epsilon \leq \lambda \leq L$ in $\Delta$. We first want to deform $\lambda|d z|^{2}$ in $\Delta$ so that the new metric $\tilde{\lambda}|d z|^{2}$ has a cone singularity at $O$. We let $r$ be the radial coordinate in $\Delta$ and $\chi$ be a smooth non-increasing function of $r$ so that $\chi=1$ for $r<\frac{1}{3}$ and $\chi=0$ for $r>\frac{2}{3}$. Let $\alpha>1$. We now let $\lambda_{\sigma}$ be the mollification of $\lambda$ by a symmetric mollifyer $\varphi_{\sigma}$ and $\lambda^{\sigma}=(\log \lambda)_{\sigma}$ where $(\log \lambda)_{\sigma}$ is the mollification of $\log \lambda$ by $\varphi_{\sigma}$. By choosing $\sigma$ sufficiently small, we assume that $\frac{\epsilon}{2} \leq \lambda^{\sigma}, \lambda_{\sigma} \leq 2 L$. Since

$$
\int \triangle \varphi_{\sigma}(|z-\xi|) \log \lambda(\xi)|d \xi|^{2} \geq-2 \kappa \int \varphi_{\sigma}(|z-\xi|) \lambda(\xi)|d \xi|^{2}
$$

$\lambda^{\sigma}$ satisfies the inequality

Let

$$
\triangle \log \lambda^{\sigma} \geq-2 \kappa \lambda_{\sigma}=-2 \kappa \frac{\lambda_{\sigma}}{\lambda^{\sigma}} \lambda^{\sigma} \geq-2 \kappa \frac{4 L}{\epsilon} \lambda^{\sigma} .
$$

$$
\tilde{\lambda}^{\sigma}=\sqrt{\left(\chi r^{\alpha}\right)^{2}+\left((1-\chi) \lambda^{\sigma}\right)^{2}} .
$$

If $z \in \Delta_{\frac{1}{3}}$ then $\tilde{\lambda}^{\sigma}=|z|^{\alpha}$ and hence $\Delta \log \tilde{\lambda}^{\sigma} \geq 0$. If $z \in \Delta-\Delta_{\frac{2}{3}}$ (where $\Delta_{r}$ is the disk of radius $r$ ) then $\tilde{\lambda}^{\sigma}=\lambda^{\sigma}$, so $\tilde{\lambda}^{\sigma}$ satisfies inequality (13). If $z \in \Delta_{\frac{5}{6}}-\Delta_{\frac{1}{6}}$, then $\tilde{\lambda}^{\sigma} \geq \max \left\{\chi|z|^{\alpha},(1-\chi) \lambda^{\sigma}\right\} \geq \max \left\{\frac{|\chi|}{6}, \frac{\epsilon|1-\chi|}{2}\right\} \geq \frac{\epsilon}{24}$.

We need the following calculation. Let $\phi, \psi$ be function in $\Delta$. Then

$$
\begin{aligned}
& ((1-t) \phi+t \psi)^{2} \triangle \log ((1-t) \phi+t \psi) \\
& =\quad((1-t) \phi+t \psi) \triangle((1-t) \phi+t \psi)-|\nabla((1-t) \phi+t \psi)|^{2} \\
& =((1-t) \phi+t \psi)\left[(1-t)\left(\phi \triangle \log \phi-\frac{|\nabla \phi|^{2}}{\phi}\right)+t\left(\psi \triangle \log \psi-\frac{|\nabla \psi|^{2}}{\psi}\right)\right] \\
& -|\nabla((1-t) \phi+t \psi)|^{2} \\
& =\quad(1-t)|\nabla \phi|^{2}\left[\frac{(1-t) \phi+t \psi}{\phi}-(1-t)\right]+t|\nabla \psi|^{2}\left[\frac{(1-t) \phi+t \psi}{\psi}-t\right] \\
& +((1-t) \phi+t \psi)[(1-t) \phi \triangle \log \phi+t \psi \triangle \log \psi] \\
& -2 t(1-t) \nabla \phi \cdot \nabla \psi \\
& =\quad t(1-t)|\nabla \phi|^{2} \frac{\psi}{\phi}-2 t(1-t) \nabla \phi \cdot \nabla \psi+t(1-t)|\nabla \psi|^{2} \frac{\phi}{\psi} \\
& ((1-t) \phi+t \psi)[(1-t) \phi \triangle \log \phi+t \psi \triangle \log \psi] \\
& \geq \quad((1-t) \phi+t \psi)[(1-t) \phi \triangle \log \phi+t \psi \triangle \log \psi] .
\end{aligned}
$$

Therefore,

$$
\triangle \log ((1-t) \phi+t \psi) \geq((1-t) \phi+t \psi)^{-1}[(1-t) \phi \triangle \log \phi+t \psi \triangle \log \psi] .
$$

Let $t=\frac{1}{2}$ in inequality (14), then we have

$$
\triangle \log (\phi+\psi) \geq(\phi+\psi)^{-1}(\phi \triangle \log \phi+\psi \triangle \log \psi)
$$


Substitute $\phi=\left(\chi r^{\alpha}\right)^{2}$ and $\psi=\left((1-\chi) \lambda^{\sigma}\right)^{2}$. Then

$$
\triangle \log \tilde{\lambda}^{\sigma} \geq\left(\tilde{\lambda}^{\sigma}\right)^{-2}\left[\left(\chi r^{\alpha}\right)^{2}\left(\triangle \log \chi r^{\alpha}\right)+\left((1-\chi) \lambda^{\sigma}\right)^{2}\left(\triangle \log (1-\chi) \lambda^{\sigma}\right)\right]
$$

Thus,

$$
\begin{aligned}
\triangle \log \tilde{\lambda}^{\sigma} & \geq\left(\tilde{\lambda}^{\sigma}\right)^{-2}\left(r^{\alpha}\left(\chi \triangle \chi-|\nabla \chi|^{2}\right)\right)+(1-\chi) \triangle \chi-|\nabla \chi|^{2}-\frac{8 \kappa L}{\epsilon} \lambda^{\sigma} \\
& \geq-\frac{4}{\epsilon^{2}}\left(\chi \triangle \chi-|\nabla \chi|^{2}\right)+(1-\chi) \triangle \chi-|\nabla \chi|^{2}-\frac{16 \kappa L^{2}}{\epsilon}
\end{aligned}
$$

Therefore, $\triangle \log \tilde{\lambda}^{\sigma} \geq-C$ where $C$ is independent of $\sigma$ and hence $\triangle \log \tilde{\lambda}^{\sigma} \geq$ $-\frac{2 C}{\epsilon} \tilde{\lambda}^{\sigma}=-2 \tilde{\kappa} \tilde{\lambda}^{\sigma}$ for $\tilde{\kappa} \geq \frac{2 L}{\epsilon}$ independently of $\sigma$. Thus,

$$
\tilde{\lambda}=\sqrt{\left(\chi r^{\alpha}\right)^{2}+((1-\chi) \lambda}
$$

satisfies

Define $\lambda_{t}^{\sigma}, \lambda_{t}$ by

$$
\triangle \log \tilde{\lambda} \geq-2 \tilde{\kappa} \tilde{\lambda} \text { weakly. }
$$

and

$$
\lambda_{t}^{\sigma}=\sqrt{(1-t)\left(\lambda^{\sigma}\right)^{2}+t\left(\tilde{\lambda}^{\sigma} \lambda^{\sigma}\right)}
$$

$$
\lambda_{t}=\sqrt{(1-t) \lambda^{2}+t(\tilde{\lambda} \lambda)}
$$

Substitute $\phi=\left(\lambda^{\sigma}\right)^{2}$ and $\psi=\tilde{\lambda}^{\sigma} \lambda^{\sigma}$ in inequality 14. Then

$$
\begin{aligned}
2\left(\lambda_{t}^{\sigma}\right)^{2} \triangle \log \lambda_{t}^{\sigma} & \geq 2(1-t)\left(\lambda^{\sigma}\right)^{2} \triangle \log \lambda^{\sigma}+t \tilde{\lambda}^{\sigma} \lambda^{\sigma} \triangle \log \tilde{\lambda}^{\sigma} \lambda^{\sigma} \\
& \geq-(1-t) \frac{8 L}{\epsilon} \kappa\left(\lambda^{\sigma}\right)^{3}-2 t \tilde{\kappa}\left(\tilde{\lambda}^{\sigma}\right)^{2} \lambda^{\sigma}-\frac{8 \kappa L t}{\epsilon} \tilde{\lambda}^{\sigma}\left(\lambda^{\sigma}\right)^{2}
\end{aligned}
$$

Assume that we have chosen $\tilde{\kappa}$ so that $\tilde{\kappa}>\frac{8 L}{\epsilon} \kappa$. Since $\lambda_{t}^{\sigma} \rightarrow \lambda^{\sigma}$ uniformly as $t \rightarrow 0$ (independently of $\sigma$ ), for sufficiently small $t$,

$$
\triangle \log \lambda_{t}^{\sigma} \geq-2 \widetilde{\kappa} \lambda_{t}^{\sigma}
$$

independently of $\sigma$. Letting $\sigma \rightarrow 0$,

$$
\triangle \log \lambda_{t} \geq-2 \tilde{\kappa} \lambda_{t} \quad \text { weakly }
$$

for sufficiently small $t$.

We let $\tilde{g}$ be the metric which is equal to $g$ in $\Sigma_{2}-\Delta$ and $\tilde{\lambda}|d z|^{2}$ in $\Delta$ and let $g_{t}$ be a family of metrics equal to $g$ in $\Sigma_{2}-\Delta$ and $\lambda_{t}|d z|^{2}$ in $\Delta$. By abuse of notation, we let $\tilde{g}$ (resp. $g_{t}$ ) the metric defined by $\tilde{g} / A(g)$ (resp. $\left(g_{t} / A\left(g_{t}\right)\right)$ where $A(\tilde{g})$ (resp. $A\left(g_{t}\right)$ ) is the area of $\tilde{g}$ (resp. $g_{t}$ ). In this way, we may asumme $A(\tilde{g}), A\left(g_{t}\right)=1$. Let $\tilde{d}$, and $d_{t}$ be the distance functions associated with $\tilde{g}$ and $g_{t}$ respectively. By choosing $\tilde{\kappa}$ sufficiently large, we see from the above argument that $\tilde{d}, d_{t} \in \mathcal{D}_{\tilde{\kappa}}$ for $t$ sufficiently small. We now claim that $\mathcal{E}_{\tilde{\kappa}}<\mathcal{E}_{\kappa}$. If $\mathcal{E}_{\tilde{\kappa}}=\mathcal{E}_{\kappa}$, then $(d, f)$ would be a minimizer for $\tilde{\kappa}$. Using the first variation argument, ${ }^{\tilde{d}} E^{f}={ }^{d} E^{f}$ and $(\tilde{d}, f)$ is also a minimizer for $\tilde{\kappa}$. Hence $f$ is an energy minimizing map for the metric $\tilde{g}$. Since $\tilde{g}$ has a cone singularity at $O$ of $\Delta$, the map $f$ collapses a vertical arc of its Hopf differential to $O$ by [Me5]. But since $f$ is an energy minimizing map of $g=\lambda|d z|^{2}$ and $\lambda \geq \epsilon>0$ in $\Delta$, by Theorem 16, $f$ is a local homeomorphism. This is a contradiction and hence $(d, f)$ cannot be a minimizer for $\tilde{\kappa}$. Therefore, $\mathcal{E}_{\tilde{\kappa}}<\mathcal{E}_{\kappa}$. Since $\kappa \mapsto \mathcal{E} \kappa$ is clearly a non-increasing function, for any $\kappa^{\prime}>\tilde{\kappa}, \mathcal{E}_{\kappa^{\prime}}<\mathcal{E}_{\kappa^{\prime}}$.

THEOREM 22. The limit of the $\operatorname{map} \kappa \mapsto \mathcal{E}_{\kappa}$ as $\kappa \rightarrow 1$ is 1 . 
Proof. Let $\epsilon>0$ be given. By Theorem 5.3.2, for every disk $\Delta \subset \Sigma_{2}$, there exists a smooth homeomorphism $h: \Sigma_{1} \rightarrow \Sigma_{2}$ so that $h$ restricted to $\Sigma_{1}-h^{-1}(\Delta)$ is a conformal map. Let $\rho$ be a smooth function so that $\int \rho \lambda_{h}|d z|^{2}=1$ and $\rho$ sufficiently small in $\Delta$ so that the energy of $h$ in $h^{-1}(\Delta)$ is less than $\epsilon$. Let $g=\rho \lambda_{h}|d z|^{2}$. Since $h$ is conformal in $\Sigma_{1}-h(\Delta)$, the energy of $h$ in $\Sigma_{1}-h^{-1}(\Delta)$ is equal to the area of $\Sigma_{2}-\Delta$ with respect to $g$. Thus,

$$
{ }^{g} E^{h}<\operatorname{Area}\left(\Sigma_{1}-\Delta, g\right)+\epsilon<1+\epsilon .
$$

Since $\rho$ is smooth, there is $\kappa$ large enough so that $g \in \mathcal{D}_{\kappa}$.

\section{References}

[Ah] L. Ahlfors. Lectures on Quasiconformal Mappings. D. Van Nostrand Company, Princeton.

[FT] A.E. Fischer and A.J. Tromba. A new proof that Teichmüller space is a cell. Trans. Amer. Math. Soc. 303 (1987), no.1, 257-262.

[GR] M. Gerstenhaber and H.E.Rauch On extremal quasiconformal mapppings I, II. Proc. N.A.S. 40 (1954), 808-812, 991-994.

[GS] M. Gromov and R. Schoen. Harmonic maps into singular spaces and p-adic superrigidity for lattices in groups of rank one. IHES Publications Math'ematiquej 76 (1992), 165-246.

[HK] W.K. Hayman and P.B. Kennedy. Subharmonic functions, vol. 1. Academic Press, London, 1976.

[Hu] A. Huber, Zum potentialtheoretischen Aspekt der Alexandrowschen Flächentheorie. Comment. Math. Helv. 34 (1960), 99-126.

$[\mathrm{J}] \quad$ J. Jost. Generalized harmonic maps between metric spaces. Geometric analysis and the calculus of variation (1996), 143-174.

[JS] J. Jost and R. Schoen. On the existence of harmonic diffeomorphims between surfaces. Inventiones Mathematica 66 (1982), 353-359.

[KS1] N. Korevaar and R. Schoen. Sobolev spaces and harmonic maps for metric space targets. Communications in Analysis and Geometry 1 (1993), 561-659.

[KS2] N. Korevaar and R. Schoen. Global existence theorem for harmonic maps to non-locally compact spaces. Communications in Analysis and Geometry 5 (1997), 333-387.

[Ku] E. Kuwert. Harmonic maps between flat surfaces with conical singularities. Mathematische Zeitschrift 221 (1996) 421-430.

[Le] M. Leite. Harmonic mappings of surfaces with respect to degenerate metrics. American Journal of Math. 110 (1988), 399-412.

[Me1] C. Mese. Minimal surfaces and conformal mappings in singular space. Ph.D. thesis, Stanford, 1996.

[Me2] C. Mese. Curvature of minimal surfaces in singular spaces. to appear in Communications in Analysis and Geometry.

[Me3] C. Mese. The structure of singular spaces of dimension 2. to appear in Manuscripta Mathematica.

[Me4] C. Mese. Some properties of minimal surfaces in singular spaces. to appear in Trans. Amer. Math. Soc.

[Me5] C. Mese. it Harmonic maps into singular surfaces with an upper curvature bound. preprint.

[Mo] C.B.Morrey. Multiple Integrals in the Calculus of Variations, Springer-Verlag, NY, 1966.

[Mi] Y. Miyahamra. On some properties of a Teichmüller mapping. TRU Math. 4 (1968), 36-43.

[Ni] I.G. Nikolaev. Solution of the Plateau problem of curvature at most K. Siberian Math. Journal. 20 (1979), 944-949.

[R] E. Reich. On the variational principle of Gerstenhaber and Rauch. Ann. Acad. Sc. Fennicae Ser. A. 10 (1985), 469-475.

[RS] E. Reich and K. Strebel. On the Gerstenhaber-Rauch principle. Israel J. Math. 57 (1987), 89-100.

[Re1] Y.G. Reshetnyak. Geometry IV Non-regular Riemann geometry. Translated by E. Primrose. Encyclopedia of Mathematical Science, 70. Springer-Berlag, Berlin, 1993.

[Re2] On the isoperimetric property of two dimensional manifolds of curvature not greater than K. Vestn. Leningr. Univ, 16 no. 19 (1961), 58-76. 
[SY] R. Schoen and S.T.Yau. On univalent harmonic maps between surfaces. Inventiones Mathematica 44 (1978), 265-278.

[Se] T. Serbinowski. Harmonic maps into metric spaces with curvature bounded above. Ph.D. thesis, University of Utah, 1995.

[T] A.J. Tromba. On the energy function for the Weil-Petersson metric on Teichmüller space. Manuscripta Math. 59 (1987), no.2, 249-260.

[Wo1] M. Wolf. Harmonic maps from surfaces to R-trees. Math. Z. 218 (1995) no.4 577-593.

[Wo2] M. Wolf. High energy degeneration of harmonic maps between surfaces and rays in Teichmüller space. Topology 30 (1991). no.4, 517-540.

[Wo3] M. Wolf. The Teichmüller theory of harmonic maps. Journal of Differential Geometry 29 (1989) no.2, 449-479.

[Y] S. Yamada. Weil-Peterson convexity of the energy functional on classical and universal Teichmüller spaces. Journal of Differential Geometry 51 (1999) no.1.

Box 5657, Department of Mathematics, Connecticut College, 270 Mohegan Ave., New London, CT 06320

E-mail address: cmes@conncoll.edu 